\title{
REFLEXIONES SOBRE LA SELECCIÓN Y PROMOCIÓN DEL PROFESORADO UNIVERSITARIO DESDE LA PERS- PECTIVA DE LA IGUALDAD DE GÉNERO
}

\author{
EVA DESDENTADO DAROCA \\ Universidad de Alcalá
}

El análisis de la selección y promoción del profesorado universitario desde la perspectiva de género es una cuestión crucial. La igualdad de hombres y mujeres es un valor social de primer orden y la Universidad no es una institución cualquiera. Es centro del saber y la cultura; punta de lanza del avance del conocimiento; y motor de la conciencia crítica de nuestra sociedad.

Resulta, pues, ineludible hacerse las siguientes preguntas: ¿es la Universidad un entorno caracterizado por la igualdad de género?, ¿favorece la igualdad entre hombres y mujeres o, por el contrario, la dificulta y perpetúa situaciones históricas de desigualdad? ¿está ya todo hecho o nos queda camino por recorrer? Y, en tal caso, ¿qué camino hay que seguir?, ¿qué se puede hacer?

Las cifras son elocuentes no sólo en España sino en general, en nuestro entorno europeo, como muestran informes como Cientificas en cifras 2015 y She Figures 2015. En ambos se pone de relieve la existencia de avances en la presencia de la mujer entre el profesorado universitario, aunque a un ritmo muy lento que no guarda correlación con la presencia de la mujer en los estudios universitarios del grado y el postgrado. Pero además la mujer está infrarrepresentada en las categorías más elevadas del profesorado universitario y en los puestos de responsabilidad de gestión académica ${ }^{1}$. En nuestro país nos encontramos por encima de la media europea en número total de mujeres científicas, pero por debajo de la media en integración vertical.

La presencia de las mujeres en cargos unipersonales de política y gestión universitaria es baja y disminuye además según se va ascendiendo en la categoría. En los rectorados se sitúa en torno a un $2 \%$.

En la Universidad de Alcalá, según datos de su Plan de Igualdad, los hombres son mayoría en el PDI, siendo el peso relativo de las mujeres del 39,6\%. Sin embargo, en el resto de los colectivos ocurre lo contrario. Así, el personal de administración y servicios (PAS) cuenta en su plantilla con un total de 491 mujeres, que suponen más del 63\%, frente a un total de 283 hombres. En los estudios de Grado, las alumnas suponen un $53,2 \%$ sobre el total de estudiantes matriculados, superando en un millar a los alumnos, y de igual manera ocurre con las alumnas de Máster que alcanzan un peso relativo del 55\%, superando a los hombres en 156 efectivos. Si se considera la totalidad del personal de la UAH (PDI y PAS), el reparto por género está muy cerca de la paridad, pues las mujeres suponen un $47,2 \%$. Sin embargo, cuando se analiza la concentración de hombres y mujeres en las dos categorías de personal hay una gran diferencia entre ambos: casi el $80 \%$ de los hombres forman parte del PDI y solo el $20 \%$ del PAS; por el contrario, del total de mujeres, el $57 \%$ pertenece al colectivo del PDI y el $43 \%$ al PAS. En definitiva, los hombres que trabajan en la Universidad de Alcalá se concentran en un porcentaje muy alto en el grupo de profesorado, mientras que las mujeres se reparten entre el profesorado y el personal de administración y servicios en proporciones muy similares. 
Las cifras son también significativas acerca de la existencia de mujeres en los niveles más elevados de la función docente e investigadora.

En los niveles inferiores, la posición de la mujer alcanza casi al 50\% (48,9\%), pero según se va ascendiendo en la carrera su presencia disminuye. En los puestos de Profesora Titular o equivalente el porcentaje no llega al 40\% (39'5) y en los de Catedrática se sitúa en torno al $20 \%{ }^{2}$.

En definitiva, la evolución académica de hombres y mujeres en la carrera universitaria reproduce los modelos clásicos de tijera en los que, según se va ascendiendo en las categorías se va produciendo una inversión en las cifras, con una mayor presencia de varones y una escasa presencia de mujeres. Se produce el efecto conocido como de cañería o tubería perforada ("leaky pipeline"): conforme se avanza en la carrera científica se produce un goteo, una pérdida que afecta de forma mucho más significativa a las mujeres que a los hombres, especialmente en los últimos escalones ${ }^{3}$.

El techo de cristal o el suelo pegajoso, elíjase cualquiera de estas expresivas imágenes, es manifiesto ${ }^{4}$.

No es fácil determinar a qué causas responde la situación, puesto que es indudable que confluyen diversos factores que operan en mayor o menor medida según los casos: el peso de la maternidad y de las cargas familiares; la falta aún de correspon-

2 En la Universidad de Alcalá la situación es la misma. En las Cátedras Universitarias, el índice de desigualdad $(0,5)$ alcanza una de las peores cifras obtenidas hasta ahora, dando prueba de que en esta categoría profesional las mujeres están infrarrepresentadas en un $50 \%$ respecto al peso que tienen en el total del PDI. El Plan de Igualdad de esta Universidad pone de relieve que "el número medio de años de carrera universitaria que se requiere para poder acceder a una plaza de este tipo es elevado y, dado que se ha comprobado que la participación femenina en el PDI disminuye conforme aumenta la edad, el bajo porcentaje de mujeres en las Cátedras Universitarias podría explicarse en parte por ello", de tal forma que "podría pensarse que esta desigualdad se corrigiera, al menos en parte, en la Cátedras de los tramos de edad más jóvenes". Sin embargo, en el Plan se afirma que "si se observa el porcentaje de mujeres en las Cátedras de Universidad por tramos de edad ocurre lo contrario, tanto en la UAH como en las Universidades Públicas españolas en general", pues "entre los Catedráticos más jóvenes la presencia mayoritaria de hombres es aún más acentuada". Es más, "la mayor edad media de los hombres frente a las mujeres en la Universidad de Alcalá y en la Universidad española en general, no parece que pueda justificar la baja participación femenina en las Cátedras de Universidad", lo que indica que "además de una posible diferencia en las preferencias de unos y otras por alcanzar este tipo de metas, resulta crucial analizar la hipótesis de que las mujeres enfrentan mayores dificultades que los hombres para poder acceder a los niveles más altos de la carrera académica universitaria".

El Informe periódico de cifras de la evaluación de la ANECA para la acreditación en los cuerpos docentes universitarios de 3 de enero de 2017 es ilustrativo. En el campo de las Ciencias Sociales y Jurídicas se han presentado más solicitudes de mujeres que de hombres para la acreditación a Profesor Titular (3.046 frente a 2.685) aunque se han evaluado positivamente más solicitudes masculinas (59'6\%) que femeninas (56'9\%). Sin embargo, para la acreditación a Catedrático, las cifras caen radicalmente (1.211 frente a 2.062$)$ y el porcentaje de evaluaciones positivas sigue siendo inferior en el caso de las mujeres (56,8\% frente a 53'8\%).

4 Vid. E. Latour, "Le plafond de verre universitaire: pour en finir avec l'illusion meritocratique et l'autocensure", Mouvement, n.55-56, 2008. También en http://www.cain.info/revuemouvements-2008-3-page-53.htm 
sabilidad; las diferencias entre hombres y mujeres en cuanto a sus objetivos vitales y su percepción sobre el poder; los estereotipos y prejuicios; los sesgos inconscientes en la valoración de los méritos ${ }^{5}$; factores varios difícilmente identificables pero relacionados con las influencias y el poder ${ }^{6}$; los propios sistemas de valoración...

Probablemente sólo un estudio interdisciplinar que abordara los aspectos sociológicos, psicológicos y jurídicos podría ofrecer una explicación completa de un fenómeno sin duda complejo.

El Derecho, el régimen jurídico de la selección y promoción del profesorado universitario, es pues sólo un factor más, pero, con todo y eso es lo que ahora me interesa señalar, es un factor relevante que puede contribuir a la igualdad o puede convertirse en un obstáculo más en el camino hacia la consecución de la misma 7 .

El jurista puede y debe, pues, preguntarse sobre el impacto de la regulación. El Derecho tiene que conformar un contexto de igualdad de oportunidades para la mujer y ello exige, en mi opinión, tratar a la mujer en el ámbito académico no igual que al hombre-porque probablemente no es igual que el hombre ni en sus percepciones, ni en sus objetivos-, sino de forma equivalente, esto es, de forma que se eliminen discriminaciones indirectas o difusas. Y para ello, en mi opinión, hay que entender la realidad de la mujer; su situación, sus dificultades singulares y sus metas en la carrera científica.

La legislación dispone que los poderes públicos deberán "remover los obstáculos que impiden a las mujeres alcanzar una presencia ... en el nivel más elevado de la función pública docente e investigadora acorde con el porcentaje que representa entre los licenciados universitarios" (Preámbulo de la Ley 4/2007) y que el personal

5 Es interesante señalar que según indica el Libro Blanco Situación de las Mujeres en la Ciencia Española, tanto en nuestro país como en los de nuestro entorno, se observa la existencia de sesgos de género inconscientes en la evaluación científica y, más concretamente, en la evaluación del mérito, la capacidad y el desempeño profesional. También se explica en el Libro como en el programa STRIDE (Science and Technology Recruiting to Improve Diversity and Excellence Committee) de la Universidad de Michigan "se identificó como dificultad principal la falta de atención y el desconocimiento de los efectos de sesgo inconscientes en el resultado de los procesos de contratación y promoción", es decir, los sesgos en la evaluación. De hecho, dentro del programa STRIDE desarrollaron unos métodos de contratación que consiguieron duplicar el número de mujeres contratadas.

6 Se ha señalado que influyen "factores difícilmente delimitables u objetivables" que sugieren la existencia de una política de promoción escasamente racionalizada y dependiente de las lógicas del poder, es decir, dependiente fundamentalmente de grupos de influencia que controlan los recursos y las oportunidades", esto es, de redes informales o de influencia, y también la importancia de los apoyos, entre otros, del propio departamento. Vid. M.J. Alonso Sánchez, "Las académicas. Profesorado universitario y género", Revista de Educación, n.328, 2002, págs. 470. Es más, incluso se ha afirmado que el problema de las mujeres académicas "es que no tienen poder, que disponen de escaso poder para canjear”. Vid. M.J.Alonso Sánchez, op.cit., pág. 471.

Con razón, el Parlamento europeo en su Resolución de 9 de septiembre de 2014 sobre la carrera de las mujeres en la ciencia y en la Universidad y la lucha contra el techo de cristal en estos ámbitos, ha urgido, a la Comisión y a los Estados miembros, a analizar la normativa existente con la finalidad de revisarla y corregirla para garantizar la igualdad de trato de hombres y mujeres. 
investigador (trabaje en Universidades u organismos públicos de investigación) tiene derecho a la igualdad de género en el desempeño de sus funciones investigadoras, así como en la contratación de personal y en el desarrollo de su carrera profesional (art. 14.1.d) de la Ley de la Ciencia).

Un sistema meritocrático, basado en la valoración de los méritos y capacidades de los candidatos, es "a priori" un sistema objetivo y neutral ${ }^{8}$ desde una perspectiva de género 9 . Es, por ello, en principio, el sistema más adecuado para alcanzar esa igualdad, aunque desde algunas posiciones se haya abogado por medidas más radicales como sacar a concurso plazas desvinculadas de los procesos de promoción con la finalidad de equilibrar la infrarrepresentación de mujeres ${ }^{10} \mathrm{o}$ incluso la introducción de cuotas para el acceso a las Cátedras; una opción controvertida y, sin duda, más que discutible en el entorno universitario si tenemos en cuenta la singular relevancia del mérito y la capacidad en este ámbito en el que ha de perseguirse, ante todo, la calidad y la excelencia ${ }^{11}$.

En cualquier caso, el que un sistema meritocrático sea el más conveniente para lograr la igualdad y romper los techos de cristal o limpiar los suelos pegajosos dependerá de diversos factores, como a quién se encomiende la tarea de evaluación, qué se compute como mérito y capacidad y cómo se valoren esos méritos y capacidades. Y lo cierto es que las cifras demuestran que los resultados no son los esperados, que las mujeres siguen teniendo más dificultades de acceso a la cumbre de la carrera académica. Algo, pues, está fallando en nuestro modelo meritocrático, más allá de que pueda también haber un cierto factor generacional.

Resulta, por tanto, imprescindible analizar cómo es el actual sistema de selección y promoción del profesorado universitario desde una perspectiva de género.

Para el acceso a los cuerpos docentes universitarios de Profesor Titular y Catedrático es necesario obtener previamente la acreditación nacional que otorga la ANECA. El acceso a los puestos de Ayudante doctor, Contratado doctor y Profesor Colaborador también exige acreditación previa.

8 Sobre los obstáculos con los que se encuentran las mujeres en sus carreras académicas pese a tratarse de un sistema meritocrático, vid. E.Escolano Zamorano, "Discriminación en un medio meritocrático: las profesoras en la Universidad española”, Revista Mexicana de Sociología, vol.68, n.2, 2006, págs.231-263.

9 Si bien, como también se ha indicado, la meritocracia puede que esté siendo, en la práctica, "el manto" que oculta las causas profundas de la desigualdad. Vid., en este sentido, F.Arranz Lozano, "Las mujeres y la Universidad española: estructuras de dominación y posición de las mujeres en el profesorado universitario", Subjetividad y Procesos Cognitivos, 2004, pág. 33.

10 J. González García, "Cuestiones de género y profesorado universitario", www.globalpoliticsandlaw.com/2017/06/02/genero-profesorado-universitario.

11 El profesorado universitario femenino no es proclive a este tipo de medidas dada la relevancia que en el ámbito investigador tiene el criterio del mérito y de la calidad y prefiere la adopción de medidas que compensen los factores que determinan su situación de desigualdad en la valoración de méritos. Vid. I. Vivas Tesón, "La investigación científica en la Universidad de Sevilla: un análisis y diagnóstico de la desigualdad de género", IV Congreso Universitario Nacional Investigación y Género, 4", Sevilla, 2012. Puede consultarse en http://idus.us.es 
La acreditación tiene por objetivo evaluar los méritos y competencias de los candidatos a estas plazas, pero la selección final y acceso al puesto en cuestión se produce a través de los correspondientes concursos públicos que se celebren en las Universidades.

Tanto en relación con las Comisiones de Acreditación como de los Tribunales que resuelven los concursos, la legislación dispone que se procurará una composición equilibrada entre mujeres y hombres, salvo que no sea posible por razones fundadas y objetivas, debidamente motivadas. La cuestión podrá ser qué haya de entenderse por razones fundadas y objetivas y, desde luego, es discutible que la escasa presencia de mujeres de la categoría correspondiente en ese sector de conocimiento pueda considerarse una razón atendible para exonerar del requisito, pues esta interpretación permitiría perpetuar la situación de desigualdad de partida contra la que precisamente quiere lucharse, pero, en fin, habrá, en todo caso, que acudir a criterios de razonabilidad y analizar la motivación y justificación ofrecidas si no se cumple con esa presencia equilibrada que exige la ley.

Con todo, y sin intención de extenderme ahora sobre este punto, lo cierto es que la eficacia de la garantía de la paridad en las comisiones no es clara e incluso podría presentar aspectos problemáticos desde la propia perspectiva de la consecución de la igualdad $^{12}$.

Más relevancia tienen, en mi opinión, los criterios de evaluación y los baremos que se utilizan en la acreditación que es el filtro que hay que superar para poder acceder a los concursos de acceso a las plazas correspondientes ${ }^{13}$. Y también la valoración que después se realice de los méritos y capacidades alegadas en el correspondiente

12 Por un lado, porque el tener que formar parte de las comisiones para cumplir con el criterio de la paridad se ha convertido en una carga para las mujeres académicas que, además, en determinados niveles no constituyen un número nutrido, con lo que la carga proporcional es mayor, sin que, por otra parte, se considere un mérito relevante ni sea una tarea especialmente remunerada. Es más, la carga y los desplazamientos que a veces lleva aparejado su cumplimiento puede terminar agravando los problemas de conciliación de la vida laboral, personal y familiar. Vid. I. Vivas Tesón, op.cit. Pero, además, tampoco debe perderse de vista otros inconvenientes sociológicos y psicológicos adicionales. Muchas de las mujeres que ocupan ahora posiciones elevadas en el mundo universitario forman parte de una élite que constituye una excepción en un mundo androcéntrico y que para llegar ahí ha hecho renuncias personales y esfuerzos especiales que no han tenido que enfrentar sus compañeros varones. En ese contexto pueden caer en el llamado "síndrome de la abeja reina", es decir, en "esa tendencia de algunas mujeres que han alcanzado altas posiciones en áreas dominadas por hombres a sentir que lo han hecho por sus propios méritos, sin ninguna consideración a su sexo" y sin ninguna predisposición "a ahorrar a las otras mujeres los esfuerzos que ellas mismas han tenido que desplegar para llegar al puesto de responsabilidad donde están, incluyendo también la tendencia a sacar gloria y benefício del hecho de ser pocas las que su sexo en su ámbito". Pautas de conducta a las que, a veces, se suma la disociación respecto de su sexo y la insolidaridad con los problemas de la mayoría de las mujeres. M.J. Alonso Sánchez, op.cit.

13 Ya en el año 2005, en el documento de trabajo de la Comisión Europea Women and Science: Excellence and Innovation- Gender Equality in Science, Bruselas, 11 de marzo de 2005, SEC(2005) 370, se señalaba que:

"Possible gender bias can occur in the characterization of scientific excellence, in the assessment criteria, in the choice of the explicit and implicit indicators for scientific excellence, in 
concurso. Me centraré en los criterios y baremos para la acreditación no sólo porque al estar fijados con carácter nacional para las plazas funcionariales, ello facilita su análisis, sino porque en gran medida terminan teniendo también una influencia en el establecimiento de los baremos para los concursos de selección y promoción.

Un análisis panorámico de los criterios de acreditación permite señalar que los mecanismos de valoración que se manejan en la actualidad distan de ser realmente neutrales desde una perspectiva de género. Algunos de ellos pueden claramente afectar de una forma más negativa a las mujeres que a los hombres.

Para realizar el análisis hay que tener en cuenta los datos estadísticos sobre la situación de la mujer en la Universidad. Así, conviene no perder de vista que la mujer ostenta con menor frecuencia puestos de gestión académica unipersonales ${ }^{14}$, que solicita menos y obtiene también menos proyectos de investigación ${ }^{15}$ y patentes, que participa con menor frecuencia en puestos de evaluación en la Administración educativa y que tiene más dificultades para la movilidad y para la realización de estancias en el extranjero especialmente si éstas son de larga duración en la medida en que sigue corriendo fundamentalmente con las tareas de cuidado en el ámbito doméstico. La mujer presenta, sin embargo, una mayor dedicación a la docencia ${ }^{16}$ y un especial énfasis en la calidad ${ }^{17}$.

El problema de las consecuencias de la maternidad es, sin duda, clave, no sólo por la diferente situación social de hombres y mujeres ante el cuidado de la prole, sino también porque las estadísticas demuestran que el impacto negativo de la tenencia de hijos se produce para las mujeres, pero no para los hombres. A estos efectos, resultan llamativos los datos que aparecen en el Libro Blanco Situación de las Mujeres en la Ciencia Española en el que se afirma que el análisis econométrico de la Encuesta de Recursos Humanos en Ciencia y Tecnología del Instituto Nacional de Estadística muestra que, en el caso de hombres y mujeres con similares caracterís-

the way the criteria are applied to men and women and the failure to integrate women in scientific networks and in the procedures through which criteria are applied to people".

14 Vid., sobre el tema, M.Tomás Folch, "Las barreras y los obstáculos en el acceso de los profesores universitarios a los cargos de gestión académica", Revista de Educación, n.350, 2009.

15 Aunque esta cifra ha ido mejorando. Vid. Cientificas en cifras 2015.

16 Existen diversos estudios que ponen de manifiesto que las mujeres académicas dedicas más tiempo a la preparación de material docentes que sus homólogos varones. Vid. S.M. Park y Keith citados por I.de Madariaga en el Libro Blanco Situación de las mujeres en la Ciencia española. La mayor valoración de la investigación frente a la docencia no es, por tanto, un criterio neutral desde la perspectiva de género. Por otra parte, la mayor dedicación docente también puede estar teniendo un impacto sobre la carrera académica de las mujeres en la medida en que tiene, lógicamente, un coste de oportunidad en una menor dedicación a otras labores como la dirección de tesis o la participación en procesos competitivos para la obtención de proyectos de investigación. Vid. B.Anghel, S. De la Rica y J.J. Dolado, "Diferencias por género en la carrera científica. Evidencia empírica", Libro Blanco Situación de las mujeres en la Ciencia española.

17 Vid. B.Anghel, S. De la Rica y J.J. Dolado, "Diferencias por género en la carrera científica. Evidencia empírica", Libro Blanco Situación de las mujeres en la Ciencia española. Según estos autores hay estudios que sugieren una preferencia de ellas por la calidad y de ellos por la cantidad. 
ticas de edad, antigüedad y productividad, los Profesores Titulares hombres tienen una probabilidad de ser promocionados a Catedráticos 2' 5 veces superior a la de las Profesoras Titulares ${ }^{18}$. Es, por tanto, imprescindible que se desarrolle un sistema que garantice que hombres y mujeres están en condiciones de igualdad a la hora de poder formar una familia y desarrollar una carrera profesional plena. La mujer no debería verse en la tesitura de tener que elegir entre la igualdad en la carrera profesional y una vida personal y familiar. A ningún hombre Catedrático se le ha exigido nunca renunciar a tener hijos; las mujeres académicas tampoco tienen por qué encontrarse obligadas a elegir entre la promoción en su carrera y la crianza de hijos ${ }^{19}$.

Es frecuente que, en los criterios de valoración de los currículos, se insista en la producción regular o lineal de resultados o que se tenga en cuenta especialmente la producción de los últimos años. Así en los documentos de la ANECA se ha venido señalando que para la acreditación a Titular se tendrán especialmente en cuenta las publicaciones de los últimos cinco años y para la Cátedra las de los últimos diez. Los sexenios de investigación como su propio nombre indica también atienden a un criterio temporal, pues se evalúan tramos de seis años.

Pues bien, los criterios de carácter estrictamente temporal como los enunciados pueden perjudicar a la mujer que, si no quiere renunciar a la vida familiar, puede necesitar organizar su carrera investigadora de una forma distinta a la masculina; no lineal ni regular, sino, por el contrario, más concentrada e intensa en determinados espacios de tiempo y más liviana en otros que coincidan con la maternidad y el cuidado de hijos de escasa edad, pues es una realidad social que las mujeres siguen cubriendo mayoritariamente estas tareas de cuidado. Su calidad y mérito investigadores y docentes no son menores por ello, ni creo que pueda afirmarse que tenga consecuencias negativas para el avance o el conocimiento científico. Téngase en cuenta que la mujer puede tener los mismos resultados de investigación que un hombre, pero distribuidos de forma distinta en el tiempo para compatibilizar el esfuerzo de la maternidad y del cuidado de hijos con el esfuerzo investigador.

Ni el mérito, ni la capacidad, ni siquiera la eficiencia en el ámbito universitario justifica el efecto adverso que este tipo de criterios temporales puede tener en las carreras universitarias de las mujere ${ }^{20} \mathrm{y}$, singularmente, en su promoción a los niveles más elevados del profesorado universitario. Y en algunos casos sería fácil introducir mecanismos de corrección que eviten las consecuencias de desigualdad que se generan. Por ejemplo, en materia de sexenios, si un sexenio se ha obtenido con una producción muy superior a la media y a la hora de solicitar el siguiente la producción es menor por razones como las anteriormente aludidas, podría permitirse solicitar una compensación que permita poner de manifiesto en una valoración conjunta de los

18 Vid. I.Sánchez de Madariaga, "Políticas de género en la Ciencia. Suprimir sesgos y promover excelencia" en el Libro Blanco Situación de las Mujeres en la Ciencia Española.

19 Según datos del Libro Blanco Situación de las Mujeres en la Ciencia Española sólo un $31 \%$ de las catedráticas tiene hijos frente a un $54 \%$ de los varones, de lo que se deduce que las mujeres sienten que para prosperar en sus carreras académicas han de renunciar al desarrollo pleno de su vida personal.

${ }^{20} Y$ del que ellas son, por cierto, plenamente conscientes. Vid. I. Vivas Tesón, op.cit. 
dos tramos arroja un resultado positivo. También podría eximirse de la exigencia de que el último sexenio esté vivo cuando ese periodo temporal haya coincidido con la maternidad $^{21}$. Y, en definitiva, cabría optar por una valoración global del currículo no condicionada por factores de linealidad temporal y/o que tenga en consideración las cargas de la maternidad y el cuidado de hijos y personas dependientes.

La insistencia en las estancias de larga duración en el extranjero y en la movilidad también puede representar un factor desfavorable para la mujer, especialmente si se combinan con el criterio temporal, pues puede que el periodo a evaluar haya coincidido con el periodo de maternidad y crianza que dificulta los cambios geográficos. Poner un peso importante en estos factores puede ser especialmente injusto además si tenemos en cuenta que la estancia en el extranjero y la movilidad no son fines en sí mismos, sino que son únicamente un instrumento para lograr la calidad, pero no el único cauce para alcanzarla. Se puede realizar una investigación de gran calidad sin necesidad de irse al extranjero, especialmente hoy en día con las posibilidades que abren las nuevas tecnologías. Valorar los resultados de la investigación y de la docencia, la calidad en ambas vertientes, más que los medios utilizados no sólo resulta más adecuado desde la perspectiva de la selección conforme al mérito y a la capacidad, sino también menos perjudicial desde la perspectiva de género.

El primer sistema de acreditación configurado por el Real Decreto 1312/2007 preveía como aspectos a valorar la investigación, la docencia, la transferencia de conocimientos, la actividad profesional y la gestión académica, de tal forma que para obtener la puntuación necesaria para lograr la acreditación era necesario conseguir puntuación en todos los campos que se presentaban como campos estancos y rígidos. Los puntos que no se lograban en alguno de ellos no eran compensables con un mayor número de puntos obtenidos en otro campo. Así, por ejemplo, si no se obtenían determinados puntos en gestión o en actividad profesional resultaba muy complicado acceder a la Cátedra, aunque se tratara de un excelente docente e investigador o aunque se tratara de un excelente investigador y profesional con menor pero buena calidad docente. De esta forma, se imponía al profesorado universitario un mismo perfil consistente en tener que dedicarse a todas las distintas vertientes del baremo con independencia de la vocación y de las posibilidades de cada uno.

El peso relativo otorgado a actividades ajenas a la docencia y a la investigación, además de discutible desde la perspectiva de la función del profesorado universitario y de la búsqueda de la calidad y eficiencia, también perjudicaban especialmente a las mujeres. No sólo porque pueden sufrir más las dificultades de la conciliación de la vida familiar y laboral y, consiguientemente, de sumar actividades profesionales o de otro tipo a la ya de por sí ardua tarea de compatibilizar tareas docentes, investigadoras y de cuidado de hijos, sino también porque, como demuestran las estadísticas, las mujeres no están accediendo en la actualidad, en porcentajes equiparables a los hombres, a los puestos de gestión y representación universitaria. Tener en cuenta estas tareas como méritos sitúa, por tanto, a las mujeres en un punto de partida más débil que a los hombres y permitirá, en consecuencia, no sólo computar una tarea

${ }^{21}$ En este sentido, J. González García, op.cit. 
que ya se retribuye adicionalmente y que tiene ventajas adicionales como la reducción de docencia, sino también ahondar las dificultades de las mujeres en el acceso a los niveles más altos de los cuerpos docentes e investigadores. Sin que, por cierto, ello esté justificado desde la perspectiva de la labor propia del profesor universitario que es primordialmente la investigación y la docencia.

La reforma de la acreditación por el Real Decreto 415/2015 ha introducido algunos cambios que, aunque no parece que hayan sido motivados por la consecución de una mayor igualdad de género, sí pueden valorarse positivamente desde esta perspectiva. Porque el Real Decreto parte de reconocer que es difícil para el profesorado universitario -y aquí cabría añadir y especialmente para las mujeres- alcanzar simultáneamente un nivel elevado en todas las facetas que se valoraban conforme al sistema anterior. Y añade que lo razonable es centrarse en los resultados obtenidos en la investigación y la docencia. De esta forma, una valoración elevada en estos campos que son los nucleares del profesorado universitario hace innecesario obtener puntuaciones en los restantes (transferencia de conocimientos, actividad profesional y gestión), aunque la nueva regulación abre la vía a distintos perfiles de tal forma que los puntos de unos y otros campos pueden compensarse.

En definitiva, los baremos aparentemente neutros pueden producir de forma inintencionada efectos adversos o favorables para lograr la igualdad de género en la selección y promoción del profesorado. Resulta esencial el análisis de las estadísticas desagregadas por razón de sexo sobre la situación, las percepciones y las metas de hombres y mujeres en el entorno universitario y también evaluar la normativa desde la perspectiva de género. A estos efectos, la normativa prevé precisamente los informes de evaluación de impacto de género. Resulta decepcionante ver como en su Dictamen 129/2015, de 7 de mayo, sobre el Real Decreto 415/2015, el Consejo de Estado señala que la memoria del Real Decreto, en cuanto al impacto por razón de género, transcribe el típico párrafo anodino en el que se señala que dada la materia que regula la norma el impacto es nulo, haciendo referencia además a una materia -la creación y reconocimiento de universidades- que ni siquiera era la que constituía el objeto de regulación.

Si queremos lograr la igualdad de las mujeres en la selección y promoción del profesorado universitario es imprescindible que el análisis desde la perspectiva de género de la regulación de los criterios de valoración se tome en serio y se utilicen debidamente las herramientas que prevé, a ese fin, el ordenamiento jurídico. Sólo así se conseguirá que el Derecho sea una herramienta que contribuya a mejorar la situación y a romper el techo de cristal. 